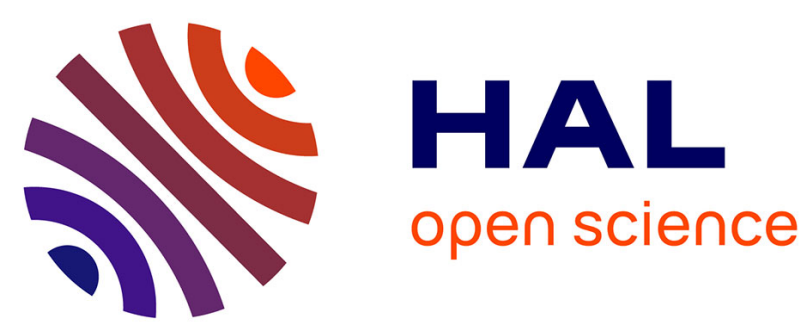

\title{
On continuous boundary time-varying feedbacks for fixed-time stabilization of coupled reaction-diffusion systems
}

Nicolás Espitia, Andrey Polyakov, Denis Efimov, Wilfrid Perruquetti

\section{- To cite this version:}

Nicolás Espitia, Andrey Polyakov, Denis Efimov, Wilfrid Perruquetti. On continuous boundary timevarying feedbacks for fixed-time stabilization of coupled reaction-diffusion systems. CDC $2018-57$ th IEEE Conference on Decision and Control, Dec 2018, Miami Beach, United States. hal-01892409

\author{
HAL Id: hal-01892409 \\ https://hal.inria.fr/hal-01892409
}

Submitted on 10 Oct 2018

HAL is a multi-disciplinary open access archive for the deposit and dissemination of scientific research documents, whether they are published or not. The documents may come from teaching and research institutions in France or abroad, or from public or private research centers.
L'archive ouverte pluridisciplinaire HAL, est destinée au dépôt et à la diffusion de documents scientifiques de niveau recherche, publiés ou non, émanant des établissements d'enseignement et de recherche français ou étrangers, des laboratoires publics ou privés. 


\section{On continuous boundary time-varying feedbacks for fixed-time stabilization of coupled reaction-diffusion systems}

\author{
Nicolás Espitia
}

Andrey Polyakov

\author{
Denis Efimov
}

\author{
Wilfrid Perruquetti
}

\begin{abstract}
This paper considers the problem of finite-time stabilization of coupled reaction-diffusion partial differential equations by means of boundary time-varying feedbacks; moreover, the time of convergence can be prescribed in the design. The design of time-varying feedbacks is carried out based on the backstepping approach. Selecting a suitable target system with time varying-coefficients, the resulting kernel of the backstepping transformation is time-varying which provides the control feedback to be time-varying as well. The target system turns out to be fixed-time stable and two cases for the control design are pointed out in order to obtain either boundedness or fixed-time convergence of the original system. A simulation example is presented to illustrate the results.
\end{abstract}

\section{INTRODUCTION}

Finite-time concepts have been subject for many years in the framework of linear and nonlinear ordinary differential equations (see e.g. [11], [7], [3], [20], [12]). The need to meet some performance, time constraints and precision has motivated the stabilization and estimation in finite time.

For partial differential equations (PDEs), the problem of finite-time stabilization and estimation has also become an attractive research area. It is known that finite-time convergence may be a natural phenomena in PDEs. For instance, some nonlinear parabolic equations may face finitetime extinction [10]. Finite-time extinction property can be also realized for a system of conservation laws as reported in [17]. Both examples are very motivating and illustrate that finite-time property can either appear naturally or can be established by means of control actions. Indeed, for hyperbolic systems, some existing results on stabilization in finite-time can be highlighted: see e.g. [1], [5] where the backstepping method is employed to design boundary controllers. For more general classes of infinite dimensional systems, we point out homogeneity arguments and switching control as in [19]. Besides this, for linear parabolic PDEs, in particular heat equations, some recent results deal with the null controllability and exact null controllability. This analysis is actually related to the finite-time stabilization problem as studied in [6] where time-varying feedbacks via the backstepping approach are studied. It is worth mentioning that backstepping method has been used as standard tool for

*This work has been partially supported by ANR Project Finite4SoS (ANR 15-CE23- 0007) and by Project 14.Z50.31.0031 of the Ministry of Education and Science of Russian Federation and Grant 08-08 of the Government of Russian Federation. N. Espitia and W. Perruquetti are with Non-A post team, Inria Lille - Nord Europe, and CRIStAL (UMRCNRS 9189), Ecole Centrale de Lille, Cité Scientifique, 59651 VilleneuvedAscq, France. A. Polyakov and D. Efimov are with Non-A post team, Inria Lille - Nord Europe, 40. av Halley, Villenueve dAscq, France, and with ITMO University, Saint-Petersburg, Russia. Corresponding author: nicolas.espitia-hoyoseinria.fr design feedback laws for PDEs. Closed-form controllers for scalar constant-parameter reaction-diffusion equations were introduced in [21]. In [2] and [16] some generalizations are presented for coupled reaction-diffusion systems with constant parameters. More recently, [25] and [9] consider a more general case of coupled reaction-diffusion systems with spatially varying coefficients. Verification of well-posedness issues of kernel transformation is also deeply studied.

Several physical systems are modeled by parabolic PDEs with relevant applications. To mention a particular one: large networks of multi-agent systems [13]. Indeed, the modeling of multi-agent systems by a continuum model may be posed in terms of diffusion parabolic equations. Furthermore, some results on finite-time deployment of multi-agent systems are reported in [14]. This motivates our work to developed finite-time concepts and control strategies for coupled linear parabolic PDEs.

This paper considers the problem of fixed-time stabilization of coupled reaction-diffusion systems. Highly inspired by [6], [18], [2] and [16], we propose continuous boundary time-varying feedbacks for finite-time stabilization in a prescribed time. Actually, this work borrows the idea of prescribed time from [23] (for normal-form nonlinear systems) by using time-varying feedbacks subject to scaling of the states with blowing up functions.

The main contribution of our work relies then on the use of boundary time-varying feedbacks obtained via the backstepping transformation; whose kernels are time-varying as well. To that end, a suitable target system is employed to come up time-varying kernels subject to suitable blowing up functions. We provide an explicit representation of kernel solutions. Two cases are pointed out regarding the choice of the blowing up function in order to obtain either boundedness or fixed-time convergence of the original system.

This paper is organized as follows. In Section II, we introduce some preliminaries on the backstepping approach and the class of parabolic system. Section III provides our approach towards fixed-time stabilization. Section IV provides a numerical example to illustrate the main results. Finally, conclusions and perspectives are given in Section V. Due to space limitations, the proofs of most of the results are omitted.

Notations: $\mathbb{R}^{+}$will denote the set of nonnegative real numbers. The set of all functions $g:[0,1] \rightarrow \mathbb{R}^{n}$ such that $\int_{0}^{1}|g(x)|^{2} d x<\infty$ is denoted by $L^{2}\left((0,1), \mathbb{R}^{n}\right)$ and is equipped with the norm $\|\cdot\|_{L^{2}\left((0,1), \mathbb{R}^{n}\right)}$. Given a real-valued square matrix $A, S[A]$ denotes its symmetric part $S[A]=$ $\left(A+A^{T}\right) / 2$ and $\sigma_{i}(A)(i=1,2, \ldots, n)$ the corresponding 
eigenvalues. $\sigma_{m}(A), \sigma_{M}(A)$ denote the smallest and largest eigenvalues, respectively. $\Gamma(\cdot)$ denotes the Gamma function. $I_{m}(\cdot), J_{m}(\cdot)$ with $m \in \mathbb{Z}$, denote the modified Bessel and (nonmodified) Bessel functions of the first kind, respectively. $L_{m}^{(\alpha)}(\cdot)$ denotes the generalized Laguerre polynomial. ${ }_{1} F_{1}(a ; b ; p)$ denotes the (Kummer) confluent hypergeometric function. Finally, $\left(\begin{array}{l}n \\ k\end{array}\right):=\frac{n !}{k !(n-k) !}, k=1,2, . ., n$ denotes the binomial coefficients.

\section{PREliminaries ON BACKSTEPPING BOUNDARY CONTROL OF COUPLED REACTION-DIFFUSION SYSTEMS}

In this section we briefly bring back some results on the backstepping boundary control of coupled reaction-diffusion systems. Consider the following unstable system [2], [16]:

$$
u_{t}(t, x)=\Theta u_{x x}(t, x)+\Lambda u(t, x)
$$

where $u: \mathbb{R}^{+} \times[0,1] \rightarrow \mathbb{R}^{n}$ is the system state with $\Theta \in$ $\mathbb{R}^{n \times n}$ is a diagonal positive matrix of the form $\Theta=\operatorname{diag}\left(\theta_{i}\right)$, $\theta_{i}>0$, for all $i=1,2 \ldots, n$. $\Lambda \in \mathbb{R}^{n \times n}$ is a real-valued matrix for coupling reaction terms.

This system is equipped with the following Dirichlet boundary condition:

$$
\begin{aligned}
& u(t, 0)=0 \\
& u(t, 1)=U(t)
\end{aligned}
$$

and initial condition:

$$
u(0, x)=u_{0}(x)
$$

where $U(t) \in \mathbb{R}^{n}$ is the control input.

It is well-known that in open loop $(U(t)=0)$, the system may not be stable due to the coupling term $\Lambda u(t, x)$ whenever $S[\Lambda]$ has positive and sufficiently large eigenvalues. In order to exponentially stabilize the system, the backstepping method is considered by using an invertible Volterra integral transformation, given as follows,

$$
w(t, x)=u(t, x)-\int_{0}^{x} K(x, y) u(t, y) d y
$$

that converts the unstable linear coupled PDE system (1)-(3) into a stable linear coupled reaction-diffusion system, usually called target system:

$$
w_{t}(t, x)=\Theta w_{x x}(t, x)-C w(t, x)
$$

with boundary conditions

$$
\begin{aligned}
& w(t, 0)=0 \\
& w(t, 1)=0
\end{aligned}
$$

and initial condition:

$$
w(0, x):=w_{0}(x)=u_{0}(x)-\int_{0}^{x} K(x, y) u_{0}(y) d y
$$

where $K(x, y)$ is a kernel matrix function in $\mathbb{R}^{n \times n}$ and $C \in$ $\mathbb{R}^{n \times n}$ is designed to guarantee arbitrarily fast exponential convergence. Hence, $K$ and $U$ are suitably chosen to realize the transformation.

\section{A. Kernel equations}

Following the standard methodology in the backstepping approach, it has been shown (see e.g. [2] for more details) that by introducing (4) into (5)-(6), using the Leibniz differentiation rule, integrating by parts and using the the boundary conditions, the original system is transformed to the target system with the kernel of the transformation (4) satisfying the following PDE matrix system:

$$
\begin{array}{r}
\Theta K_{x x}(x, y)-K_{y y}(x, y) \Theta=K(x, y) \Lambda+C K(x, y) \\
K_{y}(x, x) \Theta+\Theta K_{x}(x, x)+\Theta \frac{d}{d x} K(x, x)+\Lambda+C=0 \\
\Theta K(x, x)-K(x, x) \Theta=0 \\
K(x, 0)=0
\end{array}
$$

Kernel equations evolve in a triangular domain given by $\mathcal{T}=\left\{(x, y) \in \mathbb{R}^{2}: 0 \leq y \leq x \leq 1\right\}$. In addition, as pointed out in [2], [16], relation (11) may be fulfilled either by imposing that all coupled equations have the same diffusivity value $\theta_{i}=\theta, i=1,2, . . n$, i.e. $\Theta=\theta I_{n \times n}$; or by enforcing the kernel matrix to have the form $K(x, y)=k(x, y) I_{n \times n}$. Although the latter option allows to simplify significantly the PDE system (9)-(12) turning the problem into a scalar solvability problem (as in [21]), it brings an additional constraint on the choice of matrix $C$. Such a constraint is the following:

$$
C=-\Lambda+\gamma^{*} \Theta
$$

where $\gamma^{*}$ is a scalar parameter.

Under the first scenario (equi-diffusivity coefficients), the boundary value problem has a $C^{\infty}$ solution in the domain $\mathcal{T}$ (see [16, Theorem 3.3]), given by

$$
\begin{aligned}
K(x, y)= & -\sum_{n=0}^{\infty} \frac{\left(x^{2}-y^{2}\right)^{n}(2 y)}{n !(n+1) !}\left(\frac{1}{4 \theta}\right)^{n+1} \\
& \times \sum_{i=0}^{n}\left(\begin{array}{c}
n \\
i
\end{array}\right) C^{i}(\Lambda+C) \Lambda^{n-i}
\end{aligned}
$$

In the case of distinct-diffusivity coefficients and under (13), the boundary value problem has a $C^{\infty}$ solution in the domain $\mathcal{T}$ whose explicit representation (see [21]) is given by

$$
\begin{aligned}
& K(x, y)=k(x, y) I_{n \times n}, \\
& k(x, y)=-y \gamma^{*} \frac{I_{1}\left(\sqrt{\gamma^{*}\left(x^{2}-y^{2}\right)}\right)}{\sqrt{\gamma^{*}\left(x^{2}-y^{2}\right)}}
\end{aligned}
$$

where $I_{m}$, with $m \in \mathbb{Z}$, denotes the modified Bessel function of the first kind.

The backstepping transformation, with $x=1$, yields the boundary control function in (2) as follows

$$
U(t)=\int_{0}^{1} K(1, y) u(t, y) d y
$$

where $K$ is given either by (14) or (15) according to the two aforementioned restrictions on the diffusivity and kernel matrices, respectively. In both cases and since the transformation (4) is invertible, the boundary control input $U(t)$ exponentially stabilizes system (1)-(3) (in $L^{2}$-norm) 
with a convergence rate governed by $\sigma_{m}(S[C])$ (see [2]). Finally, without enter in full details, it is worth mentioning that kernel equations for the inverse transformation can be readily obtained by following the same procedure.

\section{TIME-VARYING FEEDBACKS AND FIXED-TIME STABILIZATION OF COUPLED REACTION-DIFFUSION SYSTEMS}

Let us consider the following coupled reaction-diffusion system:

$$
\begin{aligned}
u_{t}(t, x) & =\Theta u_{x x}(t, x)+\Lambda u(t, x) \\
u(t, 0) & =0 \\
u(t, 1) & =U(t)
\end{aligned}
$$

and initial condition:

$$
u(0, x)=u_{0}(x)
$$

where $u:[0, T) \times[0,1] \rightarrow \mathbb{R}^{n}$ is the system state with $T>0$ given, which will be called from now prescribed time. In addition, $U(t) \in \mathbb{R}^{n}$ is the control input which will be from now a time-varying feedback having the form

$$
U(t)=\mathcal{K}(t) u(t, \cdot)
$$

The operator $\mathcal{K}(t)$ is of the form

$$
\mathcal{K}(t) u(t, \cdot)=\int_{0}^{1} K(1, y, t) u(t, y) d y
$$

The existence and uniqueness of classical solutions of the coupled system (17)-(20) is assumed for initial conditions $u_{0} \in H^{2}\left((0,1), \mathbb{R}^{n}\right)$ satisfying the zero order compatibility conditions $u(0,0)=0$ and $u(0,1)=U(0)$. Without imposing compatibility condition, solutions $u \in$ $\mathcal{C}^{0}\left([0, T) ; L^{2}\left((0,1), \mathbb{R}^{n}\right)\right)$ can be understood in the weak sense (see [6]).

\section{A. Backstepping transformation and kernel equations}

We design a time-varying feedback via the backstepping approach. We aim at steering the state of the system (17)(20) to 0 in a prescribed time $T$. Inspired by some finite-time stability results for hyperbolic PDE systems e.g. [5], [1], [8], where finite-time convergence is achieved by choosing suitable target systems; in this work, we will slightly modify the target system (5)-(6) in order to have convergence in the prescribed time $T$.

The key ingredient in this framework is the use of timevarying feedbacks. Consequently, the invertible Volterra integral transformation is chosen to depend on time. It is given as follows,

$$
w(t, x)=u(t, x)-\int_{0}^{x} K(x, y, t) u(t, y) d y
$$

rendering the kernel time-varying. The aim is to transform the system (17)-(20) into the following target system:

$$
\begin{aligned}
w_{t}(t, x) & =\Theta w_{x x}(t, x)-C(t) w(t, x) \\
w(t, 0) & =0 \\
w(t, 1) & =0
\end{aligned}
$$

with initial condition:

$$
w_{0}(x)=u_{0}(x)-\int_{0}^{x} K(x, y, 0) u_{0}(y) d y
$$

Note that different to system (5)-(6), we have now that in the right-hand side of this new target system, there is a time-dependent matrix $C(t)$ to be designed.

Following the standard method in the backstepping approach, it can be obtained that the kernel of the transformation (23) must satisfy the following PDE matrix system

$$
\begin{array}{r}
\Theta K_{x x}(x, y, t)-K_{y y}(x, y, t) \Theta=K_{t}(x, y, t) \\
+K(x, y, t) \Lambda+C(t) K(x, y, t) \\
K_{y}(x, x, t) \Theta+\Theta K_{x}(x, x, t)+\Theta \frac{d}{d x} K(x, x, t) \\
=-(\Lambda+C(t)) \\
\Theta K(x, x, t)-K(x, x, t) \Theta=0 \\
K(x, 0, t)=0
\end{array}
$$

defined on the domain $\mathcal{T}=\left\{(x, y, t) \in \mathbb{R}^{2} \times[0, T): 0 \leq\right.$ $y \leq x \leq 1\}$.

Remark 1: It is worth noticing that the right-hand side of (28) contains the time derivative of the kernel. The scalarcounterpart is similar to the one found in [22]; and in more general cases with space- and time-varying reaction parameter, one could follow [15], [26] and [4] for wellposedness issues.

The finite-time stabilization problem is now related to the problem of solvability of kernel equations (28)-(31). Solving them and choosing $U(t)$ given in (22) we realize the backstepping transformation.

Proposition 1: Let $\gamma:[0, T) \rightarrow(0, \infty)$ and $k: \mathcal{T} \rightarrow \mathbb{R}$ satisfying the following scalar PDE:

$$
\begin{aligned}
k_{x x}(x, y, t)-k_{y y}(x, y, t) & =\gamma(t) k(x, y, t)+\frac{1}{\theta} k_{t}(x, y, t) \\
k(x, 0, t) & =0 \\
\frac{d}{d x} k(x, x, t) & =-\frac{1}{2} \gamma(t)
\end{aligned}
$$

If $\Theta=\theta I_{n \times n}$, then the kernel matrix $K(x, y, t)=$ $k(x, y, t) I_{n \times n}$, satisfies (28)-(31) provided that

$$
C(t)=-\Lambda+\gamma(t) \Theta
$$

Proof: It follows the same arguments of [2, Section 4]. Imposing (35) is instrumental for $K(x, y, t)$ to meet the critical constraint (30).

From (35), only the diagonal of $C(t)$ contains time-varying functions. As we will see, such a choice along with a suitable characterization of $\gamma$ will allow us the achievement of finitetime stability result.

Remark 2: Note that we have assumed the equidiffusivity coefficients case and we have imposed a particular restriction on the structure of the kernel. This is in fact with the aim to obtain closed-form solutions of the time varyingkernel which will allow an easier numerical tractability and 
moreover, a qualitative analysis for obtaining bounds of the kernel of the backstepping transformation. Nevertheless, it is clear that the equi-diffusivity assumption is a strong limitation. A generalization with distinct diffusivity coefficients and even space-varying coefficients is much more involved and requires further analysis in order to solve numerically the kernel equations.

\section{B. Solution of the PDE kernel}

Let us choose $\gamma$ in Proposition 1 to be the solution that satisfies the following scalar nonlinear ordinary differential equation:

$$
\dot{\gamma}(t)=\frac{1+\epsilon}{\gamma_{0} T} \gamma^{(2+\epsilon) /(1+\epsilon)}(t), \quad \gamma(0)=\gamma_{0}>0
$$

where $\epsilon \geq 0$ is a design parameter, $T>0$ is given and is going to be the prescribed time. It is straightforward to verify that the solution to (36) is as follows:

$$
\gamma(t)=\frac{\left(\gamma_{0} T\right)^{1+\epsilon}}{(T-t)^{1+\epsilon}}
$$

This solution is monotonically increasing and blows up at time $T$.

Lemma 1: Let $T>0$ be given. Under assumptions of Proposition 1, and $\gamma$ satisfying (36) with $\epsilon=0$, the problem (28)-(31) has a well-posed $C^{\infty}$ solution on $\mathcal{T}$, given by

$$
\begin{aligned}
K(x, y, t) & =k(x, y, t) I_{n \times n}, \\
k(x, y, t) & =-\frac{y}{2} \frac{\gamma(t)}{\Gamma(N+1)} \sum_{n=0}^{\infty} \frac{\left(\gamma(t)\left(x^{2}-y^{2}\right)\right)^{n} \Gamma(N+n+1)}{4^{n} n !(n+1) !(N)^{n}}
\end{aligned}
$$

with $N=T \gamma_{0} \theta$. Moreover, it can be reformulated as follows:

$$
k(x, y, t)=-\frac{y}{2} \gamma(t) e^{\frac{\gamma(t)\left(x^{2}-y^{2}\right)}{4 N}} \frac{1}{N} L_{N-1}^{(1)}\left(-\frac{\gamma(t)\left(x^{2}-y^{2}\right)}{4 N}\right)
$$

where $L_{N-1}^{(1)}(\cdot)$ denotes a generalized Laguerre polynomial.

Proof: The proof essentially relies on [22, Section 5] to obtain the power series solution (38). In order to obtain (39), let us first briefly introduce the following Kummer confluent hypergeometric function, a relevant property and its connection with the generalized Laguerre polynomials (see e.g. [24] for more details). The (Kummer) confluent hypergeometric function ${ }_{1} F_{1}(a ; b ; p)$ is defined as follows:

$$
{ }_{1} F_{1}(a ; b ; p)=\sum_{k=0}^{\infty} \frac{(a)_{k}}{(b)_{k}} \frac{p^{k}}{k !}
$$

where $(a)_{k}:=a(a+1)(a+2) \cdots(a+k-1)=\frac{\Gamma(a+k)}{\Gamma(a)}$ denotes the Pochhammer symbol or rising factorial. The Kummer function has the following property:

$$
e^{p}{ }_{1} F_{1}(a ; b ;-p)={ }_{1} F_{1}(b-a ; b ; p)
$$

The generalized Laguerre polynomial expressed in terms of (40) is

$$
L_{k}^{(\alpha)}(p)=\left(\begin{array}{c}
k+\alpha \\
k
\end{array}\right){ }_{1} F_{1}(-k ; \alpha+1 ; p)
$$

Having established that, from (38) it is sufficient to set $p=$ $\frac{\gamma(t)\left(x^{2}-y^{2}\right)}{4 N}, a=N+1$ and $b=2$ in (40). In addition, note that $(n+1) !=\Gamma(n+2)$. Hence, $(38)$ is rewritten as follows

$$
k(x, y, t)=-\frac{y}{2} \gamma(t)_{1} F_{1}\left(N+1 ; 2 ; \frac{\gamma(t)\left(x^{2}-y^{2}\right)}{4 N}\right)
$$

In light of (41), we get

$k(x, y, t)=-\frac{y}{2} \gamma(t) e^{\frac{\gamma(t)\left(x^{2}-y^{2}\right)}{4 N}}{ }_{1} F_{1}\left(1-N ; 2 ;-\frac{\gamma(t)\left(x^{2}-y^{2}\right)}{4 N}\right)$

Finally, using (42) and by knowing that $\left(\begin{array}{c}(N-1)+\alpha \\ N-1\end{array}\right)=$ $\frac{\Gamma((N-1)+\alpha+1)}{\Gamma((N-1)+1) \Gamma(\alpha+1)}=N$ with $\alpha=1$, then

$$
k(x, y, t)=-\frac{y}{2} \gamma(t) e^{\frac{\gamma(t)\left(x^{2}-y^{2}\right)}{4 N}} \frac{1}{N} L_{N-1}^{(1)}\left(-\frac{\gamma(t)\left(x^{2}-y^{2}\right)}{4 N}\right)
$$

Corollary 1: If $\gamma_{0}$ and $T$ are selected such that $N=1$ (in Lemma 1), then the problem (28)-(31) has a well-posed $C^{\infty}$ solution on $\mathcal{T}$, given by

$$
\begin{aligned}
K(x, y, t) & =k(x, y, t) I_{n \times n}, \\
k(x, y, t) & =-\frac{y}{2} \gamma(t) e^{\frac{\gamma(t)\left(x^{2}-y^{2}\right)}{4}}
\end{aligned}
$$

We provide in the sequel a closed-form solution under a blowing up function (36) with $\epsilon=1$. This choice is adequate to obtain the fixed-time stability result as it is going to be stated later on.

Theorem 1: Let $T>0$ be given. Under assumptions of Proposition 1, and $\gamma$ satisfying (36) with $\epsilon=1$, the problem (28)-(31) has a well-posed $C^{\infty}$ solution on $\mathcal{T}$, given by

$$
\begin{aligned}
& K(x, y, t)=k(x, y, t) I_{n \times n}, \\
& k(x, y, t)=-\frac{y}{2} \gamma(t) \sum_{n=0}^{\infty} \frac{\left(\sqrt{\gamma(t)}\left(x^{2}-y^{2}\right)\right)^{n}}{4^{n}\left(T \gamma_{0} \theta\right)^{n}(n+1) !} \\
& \quad \times L_{n}^{(1)}\left(-\left(T \gamma_{0} \theta\right) \sqrt{\gamma(t)}\right)
\end{aligned}
$$

C. Inverse transformation and kernel equations

The inverse transformation is given by

$$
u(t, x)=w(t, x)+\int_{0}^{x} L(x, y, t) w(t, y) d y
$$

whose kernel $L(x, y, t)$ satisfies the following PDE system

$$
\begin{array}{r}
\Theta L_{x x}(x, y, t)-L_{y y}(x, y, t) \Theta=L_{t}(x, y, t) \\
-\Lambda L(x, y, t)-L(x, y, t) C(t) \\
L_{y}(x, x, t) \Theta+\Theta L_{x}(x, x, t)+\Theta \frac{d}{d x} L(x, x, t) \\
=-(\Lambda+C(t)) \\
L(x, x, t) \Theta-\Theta L(x, x, t)=0 \\
L(x, 0, t)=0
\end{array}
$$

defined on the domain $\mathcal{T}=\left\{(x, y, t) \in \mathbb{R}^{2} \times[0, T): 0 \leq\right.$ $y \leq x \leq 1\}$.

The time-varying feedback (21) can be equivalently written under the following form

$$
U(t)=\mathcal{L}(t) w(t, \cdot)
$$


The operator $\mathcal{L}(t)$ is of the form

$$
\mathcal{L}(t) w(t, \cdot)=\int_{0}^{1} L(1, y, t) w(t, y) d y
$$

Proposition 2: Let $\gamma:[0, T) \rightarrow(0, \infty)$ and $l: \mathcal{T} \rightarrow \mathbb{R}$ satisfying the following scalar PDE:

$$
\begin{aligned}
l_{x x}(x, y, t)-l_{y y}(x, y, t) & =-\gamma(t) l(x, y, t)+\frac{1}{\theta} l_{t}(x, y, t) \\
l(x, 0, t) & =0 \\
\frac{d}{d x} l(x, x, t) & =-\frac{1}{2} \gamma(t)
\end{aligned}
$$

If $\Theta=\theta I_{n \times n}$, then the kernel matrix $L(x, y, t)=$ $l(x, y, t)) I_{n \times n}$, satisfies (47)-(50) provided that

$$
C(t)=-\Lambda+\gamma(t) \Theta
$$

\section{Solution of the PDE kernel}

Lemma 2: Let $T>0$ be given. Under assumptions of Proposition 2, and $\gamma$ satisfying (36) with $\epsilon=0$, the problem (47)-(50) has a well-posed $C^{\infty}$ solution on $\mathcal{T}$, given by

$$
\begin{aligned}
L(x, y, t) & =l(x, y, t) I_{n \times n}, \\
l(x, y, t) & =-\frac{y}{2} \gamma(t) \sum_{n=0}^{\infty}(-1)^{n} \frac{\left(\gamma(t)\left(x^{2}-y^{2}\right)\right)^{n} \prod_{i=1}^{n}(N-i)}{4^{n} n !(n+1) !(N)^{n}}
\end{aligned}
$$

with $N=T \gamma_{0} \theta$. Moreover, it can be reformulated as follows:

$$
l(x, y, t)=-\frac{y}{2} \gamma(t) \frac{1}{N} L_{N-1}^{(1)}\left(\frac{\gamma(t)\left(x^{2}-y^{2}\right)}{4 N}\right)
$$

Proof: The proof follows the same reasoning as in the proof of Lemma 1.

Corollary 2: If $\gamma_{0}$ and $T$ are selected such that $N=1$ (in Lemma 2), then the problem (47)-(50) has a well-posed $C^{\infty}$ solution on $\mathcal{T}$, given by

$$
\begin{aligned}
L(x, y, t) & =l(x, y, t) I_{n \times n}, \\
l(x, y, t) & =-\frac{y}{2} \gamma(t)
\end{aligned}
$$

Theorem 2: Let $T>0$ be given. Under assumptions of Proposition 2, and $\gamma$ satisfying (36) with $\epsilon=1$, the problem (47)-(50) has a well-posed $C^{\infty}$ solution on $\mathcal{T}$, given by

$$
\begin{gathered}
L(x, y, t)=l(x, y, t) I_{n \times n}, \\
l(x, y, t)=-\frac{y}{2} \gamma(t) \sum_{n=0}^{\infty} \frac{\left(\sqrt{\gamma(t)}\left(x^{2}-y^{2}\right)\right)^{n}}{4^{n}\left(T \gamma_{0} \theta\right)^{n}(n+1) !} \\
\quad \times L_{n}^{(1)}\left(T \gamma_{0} \theta \sqrt{\gamma(t)}\right)
\end{gathered}
$$

\section{E. Fixed-time control}

The next lemma presents the condition to be fulfilled while choosing $\gamma_{0}$ in order to guarantee that the matrix $S[C(t)]:=$ $\left(C(t)+C^{T}(t)\right) / 2$ is positive definite, for all $t \in[0, T)$. This is in fact a less conservative condition than the one given in [2] which would correspond, in our case, to the positive definiteness of $S[C(0)]$.

Lemma 3: Let $C(t)$ be given by (35). If $\gamma_{0}>0$ is such that

$$
\gamma_{0}>\frac{\sigma_{M}(S[\Lambda])}{\theta}
$$

then for any $t \in[0, T)$, it holds that

$$
S[C(t)]>0
$$

The following result just allows to state the boundedness of the $L^{2}$-norm of the closed-loop system (17)-(20) when the degree of the blowing up function is set as $\epsilon=0$ and we restrict $N=1$. In such a case the controller is given by (21) and kernel given by (44). For $N \neq 1$, no conclusion on the convergence can be provided.

Proposition 3: Let $\gamma_{0}, T>0$ be given. If $\gamma_{0}$ is such that condition (61) is satisfied and $N=1$, then for the closedloop system (17)-(20), there exist $M>0$ such that for for any initial condition $u_{0} \in L^{2}((0,1), \mathbb{R})$, it holds, for all $t \in[0, T)$,

$$
\|u(t, \cdot)\|_{L^{2}\left((0,1), \mathbb{R}^{n}\right)} \leq M\left\|u_{0}\right\|_{L^{2}\left((0,1), \mathbb{R}^{n}\right)}
$$

Let us state the main result of the paper for fixed-time stabilization. The choice of the blowing up function has been instrumental for this result (i.e. with $\epsilon=1$ ). As aforementioned, with $\epsilon=0$, one may not guarantee fixedtime convergence.

Theorem 3: Let $\gamma_{0}, T>0$ be given. If $\gamma_{0}$ is such that condition (61) (in Lemma 3) holds and

$$
T \gamma_{0} \theta>\frac{1}{2}
$$

then, the time-varying feedback

$$
U(t)=\int_{0}^{1} K(1, y, t) u(t, y) d y
$$

with $K(1, y, t)$ as in (45) (at $x=1)$, stabilizes the system (17)-(20) in a prescribed $T$, i.e. for any initial condition $u_{0} \in L^{2}\left((0,1), \mathbb{R}^{n}\right)$, it holds

$$
\|u(t, \cdot)\|_{L^{2}\left((0,1), \mathbb{R}^{n}\right)} \rightarrow 0 \quad \text { as } \quad t \rightarrow T
$$

Moreover, $U(t)$ remains bounded and $|U(t)| \rightarrow 0$ as $t \rightarrow T$.

\section{Simulations}

We illustrate the results of Section III by considering a linear system of $3 \times 3$ reaction diffusion system with $\Theta=I_{n \times n}$, $\Lambda=\left(\begin{array}{lll}2 & 2 & 3 \\ 4 & 5 & 3 \\ 2 & 5 & 3\end{array}\right)$ and initial conditions $u_{1}(0, x)=20.25 x(1-x)$, $u_{2}(0, x)=10.25 x(1-x), u_{3}(0, x)=5.25 x(1-x)$ satisfying the zero order compatibility conditions. Note that, in open loop (e.g. $U(t)=0$ ), the system is unstable.

We select the blowing up function (36) with $\epsilon=1$. The parameter $\gamma_{0}$ is chosen to be $\gamma_{0}=3$. The selected prescribed time is $T=1$. Hence condition (64) holds and Theorem 3 applies. Figure 1 shows the time evolution of $L^{2}$ - norm of the closed-loop system plotted in logarithmic scale to better illustrate that with time-varying feedbacks the closed-loop system converges in a prescribe time given by $T=1$. It can be observed that the convergence to zero is faster than using linear control for exponential stabilization (red-dashed line).

Figure 2 shows the time evolution of the components of the control function $U(t)$ (65) with kernel gains (45). 


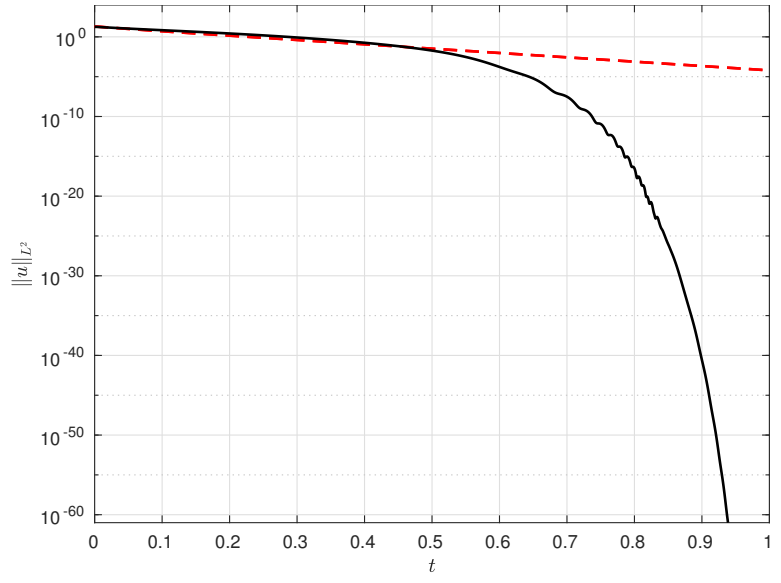

Fig. 1. Evolution of the $L^{2}$-norm of the closed-loop system (logarithmic scale) with time-varying feedback (black line) for a prescribed time $T=1$ and linear control feedback (red dashed line) for exponential stabilization.

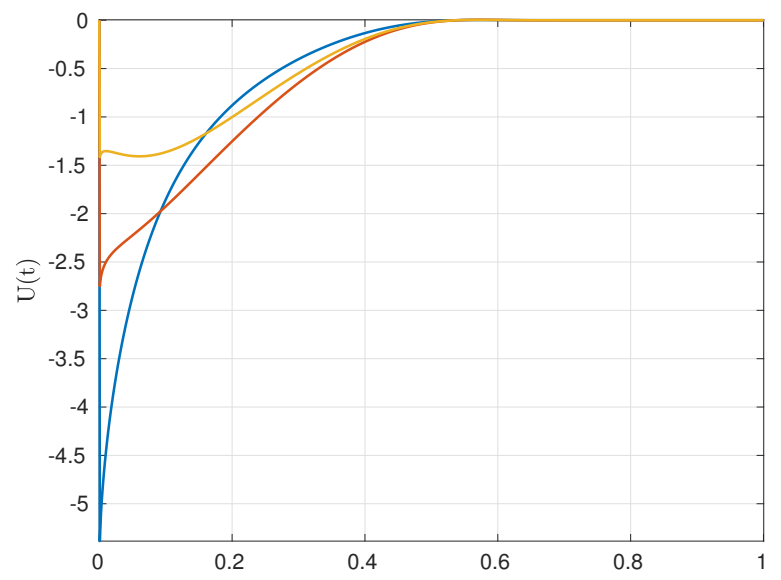

Fig. 2. Evolution of the control function $U(t)$.

\section{CONCLUSION}

In this paper, we addressed continuous boundary timevarying feedbacks towards fixed-time stabilization of coupled reaction-diffusion systems. Closed-form kernels have been obtained and under a suitable blowing up function, it is possible to obtain a time-varying feedback stabilizing the closed-loop system in a fixed-time.

A future direction line is dealing with solution approximations to time-varying kernel equations without imposing structure on the kernels nor equi-diffuivity assumption as we have done in this work and furthermore, with space varying coefficients. It is going definitely be inspired by recent works [25], [9]. Finally, fixed-time observers for coupled reactiondiffusion equations, following e.g. [16] is currently under investigation.

\section{REFERENCES}

[1] J. Auriol and F. Di Meglio. Minimum time control of heterodirectional linear coupled hyperbolic pdes. Automatica, 71:300-307, 2016.

[2] A. Baccoli, A. Pisano, and Y. Orlov. Boundary control of coupled reactiondiffusion processes with constant parameters. Automatica, 54:80-90, 2015.
[3] S.P. Bhat and D.S. Bernstein. Finite time stability of continuous autonomous systems. SIAM J. Control Optim., 38(3):751-766, 2000.

[4] D. Colton. The solution of initial-boundary value problems for parabolic equations by the method of integral operators. Journal of Differential Equations, 26(2):181 - 190, 1977.

[5] J.-M. Coron, L. Hu, and G. Olive. Finite-time boundary stabilization of general linear hyperbolic balance laws via fredholm backstepping transformation. Automatica, 84:95-100, 2017.

[6] J.-M. Coron and H.-M. Nguyen. Null controllability and finite time stabilization for the heat equations with variable coefficients in space in one dimension via backstepping approach. Archive for Rational Mechanics and Analysis, 225(3):993-1023, Sep 2017.

[7] J.-M. Coron and L. Praly. Adding an integrator for the stabilization problem. Systems \& Control Letters, 17(2):89-104, 1991

[8] J.-M. Coron, R. Vazquez, M. Krstic, and G. Bastin. Local exponential $H^{2}$ stabilization of a $2 \times 2$ quasilinear hyperbolic system using backstepping. SIAM Journal on Control and Optimization, 51(3):20052035, 2013.

[9] J. Deutscher and S. Kerschbaum. Backstepping control of coupled linear parabolic PIDEs with spatially-varying coefficients. IEEE Transactions on Automatic Control, PP(99):1-1, 2018.

[10] V.A. Galaktionov and J.L Vasquez. Necessary and sufficient conditions for complete blow-up and extinction for one-dimensional quasilinear heat equation. Archive for Rational Mechanics and Analysis, 129(3):225-244, 1995.

[11] V.T. Haimo. Finite time controllers. SIAM Journal of Control and Optimization, 24(4):760-770, 1986.

[12] F. Lopez-Ramirez, A. Polyakov, D. Efimov, and W. Perruquetti. Finite-time and fixed-time observer design: Implicit lyapunov function approach. Automatica, 87:52 - 60, 2018.

[13] T. Meurer. Some perspectives in PDE control. In Proc. 20th IFAC World Congress, Toulouse (F), July 09-14 2017.

[14] T. Meurer and M. Krstic. Finite-time multi-agent deployment: A nonlinear PDE motion planning approach. Automatica, 47(11):2534 2542,2011

[15] T. Meurer and A. Kugi. Tracking control for boundary controlled parabolic PDEs with varying parameters: Combining backstepping and differential flatness. Automatica, 45(5):1182-1194, 2009.

[16] Y. Orlov, A. Pisano, A. Pilloni, and E. Usai. Output feedback stabilization of coupled reaction-diffusion processes with constant parameters. SIAM Journal on Control and Optimization, 55(6):4112$4155,2017$.

[17] V. Perrollaz and L. Rosier. Finite-time stabilization of $2 \times 2$ hyperbolic systems on tree-shaped networks. SIAM Journal on Control and Optimization, 52(1):143-163, 2014.

[18] A. Polyakov, J-M. Coron, and L. Rosier. On boundary finite-time feedback control for heat equation. In 20th IFAC World Congress, Toulouse, France, July 2017.

[19] A. Polyakov, J.-M. Coron, and L. Rosier. On Homogeneous FiniteTime Control For Evaluation Equation in Hilbert Space. IEEE Transactions on Automatic Control, to appear, PP(99):1-1, 2017.

[20] A. Polyakov, D. Efimov, and W. Perruquetti. Finite-time and Fixedtime Stabilization: Implicit Lyapunov Function Approach. Automatica, 51(1):332-340, 2015

[21] A. Smyshlyaev and M. Krstic. Closed-form boundary state feedbacks for a class of 1-d partial integro-differential equations. IEEE Transactions on Automatic Control, 49(12):2185-2202, Dec 2004.

[22] A. Smyshlyaev and M. Krstic. On control design for pdes with space-dependent diffusivity or time-dependent reactivity. Automatica, 41(9):1601 - 1608, 2005.

[23] Y.-D. Song, Y.-J. Wang, J.-C. Holloway, and M. Krstic. Timevarying feedback for regulation of normal-form nonlinear systems in prescribed finite time. Automatica, 83:243 - 251, 2017.

[24] G. Szego. Orthogonal polynomials, volume 23. American Mathematical Society Providence, Rhode Island, 4th edition, 1975

[25] R. Vazquez and M. Krstic. Boundary control of coupled reactionadvection-diffusion systems with spatially-varying coefficients. IEEE Transactions on Automatic Control, 62(4):2026-2033, April 2017.

[26] R. Vazquez, E. Trlat, and J.-M. Coron. Control for fast and stable laminar-to-high-reynolds-numbers transfer in a 2D Navier-Stokes channel flow. Discrete \& Continuous Dynamical Systems - B, 10(4):925-956, 2008 